\title{
Effect of Selected Vestibular Exercises on Postural Stability in Elderly Women with Type 2 Diabetes
}

\author{
Kumar Goothy SS ${ }^{*}$ Ravikanth Manyam² and Arun H S Kumar ${ }^{3}$ \\ ${ }^{1}$ Department of Physiology, Vishnu Dental College, India \\ ${ }^{2}$ Department of Oral pathology, Vishnu Dental College, India \\ ${ }^{3}$ Department of Stemcology, University College Dublin, Ireland
}

*Corresponding author: Dr Sai Sailesh Kumar Goothy, Assistant professor \& Head of

\begin{tabular}{c} 
Research Article \\
Volume 4 Issue 2 \\
Received Date: March 23, 2019 \\
Published Date: April 12, 2019 \\
DoI: $10.23880 /$ ijbp-16000154 \\
\hline
\end{tabular}
the Department (I/C), Department of Physiology, Vishnu Dental College, Bhimavaram, West Godavari District, Andhra Pradesh, India, Email: dr.saisailesh@gmail.com

\section{Abstract}

Objective: The present study aimed to observe the effectiveness of vestibular exercises on postural stability in elderly women with type 2 diabetes.

Materials and methods: 30 elderly women with type-2 diabetes were recruited for the study after written informed consent by convenient sampling. Participants acted as self-controls. Vestibular exercises comprises of 45 minutes one session. Two sessions per week was administered to the participants for a period of one month. Postural stability was assed using Berg balance scale. which is a standard questionnaire to assess the postural stability.

Results: There was significant increase in the Breg Balance Scale score followed by the vestibular exercises $(\mathrm{P}<0.001)$.

Conclusion: The present study provides further evidence for the beneficial effect of practicing the selected vestibular exercises in the management of postural stability in the elderly women with type 2 diabetes. The study also recommends further detailed studies in this area to recommend implementation of vestibular exercises in the management of postural stability in type 2 diabetic patients in general.

Keywords: Vestibular exercise; Postural stability; Elderly women; Diabetes

\section{Introduction}

Vision, audition, olfaction, gustation and touch are the five special senses described by Aristotle. Vestibular apparatus is a membranous structure located within the bony labyrinth of the inner ear. It comprises of three semicircular canals and two otolith organs on either side.
These structures are filled with endolymph and are surrounded by perilymph. Semicircular canal includes horizontal, superior, and posterior canals and otolith organs include utricle and the saccule. Changes in the rate of linear motion are detected by otolith organs and rotatory or angular acceleration or deceleration are detected by semicircular canals. Vestibular system also 


\section{International Journal of Biochemistry \& Physiology}

contributes to a wide range of functions from level of reflexes to level of cognition and coordination. Hence, vestibular sense is also called as sixth sense [1]. Vestibular system is one among the first cranial cavity structures to develop in fetal life and plays an indispensable role in regular activities [2]. Human vestibular system consists of 18,500 vestibular nerve fibers, which are extensively connected with cortical and subcortical brain structures. Basic function of vestibular apparatus is to maintain equilibrium that is sense of body orientation and motion in the gravitational field [3]. Vestibular stimulation improves the quality of sleep, decreases pain, improves motor skills, developmental milestones, decreases stress, modulates and resets normal respiratory pattern, increases neural stability, improves spatial and verbal memory, modulates mood states, regulates autonomic functions and influence many other brain physiology [4,5]. Further, vestibular stimulation approach is used for management of pain disorders, psychiatric disorders, post lesional disorders and other neurological disorders [6]. Earlier researchers suggested that over stimulation of vestibular system should be avoided and under stimulation may not have any effect. So the participants should be allowed to determine their own speed or intensity of the stimulation [7]. Vestibular stimulation integrates the cortical and subcortical structures and regulates the posture. Further, through the vestibule-spinal pathways the postural stability is maintained by vestibular stimulation [8-11]. The present study aimed to observe the effectiveness of vestibular exercises on postural stability in elderly women with type 2 diabetes.

\section{Materials and Methods}

Study Setting: The present study was conducted at Department of Physiology, Vishnu Dental College, Bhimavaram.

Study Design: Pre and post without control design. The participants acted as self-controls. After recording the baseline values, vestibular exercises were practiced by the participants for one month. Post values were recorded after one month.

Study Participants: 30 elderly women with type-2 diabetes were recruited for the study after written informed consent by convenient sampling. Participants acted as self-controls. The following criteria were followed while selecting the participants for the intervention group.
Inclusion Criteria: Elderly women with type 2 diabetes and able to walk for minimum three minutes without any support (minimum 30 steps) and those without any cognitive impairment and willing to participate were included in the study.

Exclusion Ccriteria: Those with severe medical complications and vestibular disorders were excluded from the study.

Vestibular Exercises: Each session of exercises comprises of 45 minutes [12]. Two sessions per week was administered to the participants for a period of one month. The exercise comprises of three steps.

\section{Step One}

The participants were asked to lie down on their back, the participants shifted to lying on their right side, then to their left side. This cycle was repeated for ten times. Following this 3 minutes rest was given while lying on right side. Again the process was repeated and this time during rest the participant lies to his left side for 3 minutes. Then same procedure was also performed with closed eyes.

\section{Step Two}

The participant was asked to sit with legs extended straight and bend alternatively to right and left sides while the arms resting on the mat behind the body. After ten times, three minutes rest was given. During these three minutes the participants was in side-bend position to right side. After the rest, the participants repeated the side bends for ten times and again three minutes rest was given. During this three minutes rest, the participant was in side-bend position to left side.

\section{Step Three}

The participants were in sitting position and asked to move head up and down directions slowly and gently while eyes are closed. This was performed for ten times and followed by three minutes rest. Then the participant's moved his head to sideways for ten times slowly and gently while eyes are closed.

\section{Outcome Measures}

Assessment of Postural stability: Postural stability was assed using Berg balance scale [13] which is a standard questionnaire to assess the postural stability.

Ethical consideration: The present study was approved by institutional Research Committee. Informed consent 


\section{International Journal of Biochemistry \& Physiology}

was obtained from all the participants. Confidentiality of the data was maintained.

Data analysis: Data was analyzed using SPSS 20.0. Paired $t$ test was used to observe the difference between the groups. Probability value less than 0.05 was considered as significant.

\section{Results}

Figure 1 presents the demographic data of the participants and Breg Balance Scale score of the participants. There was significant increase in the Breg Balance Scale score followed by the vestibular exercises $(\mathrm{P}<0.001)$.
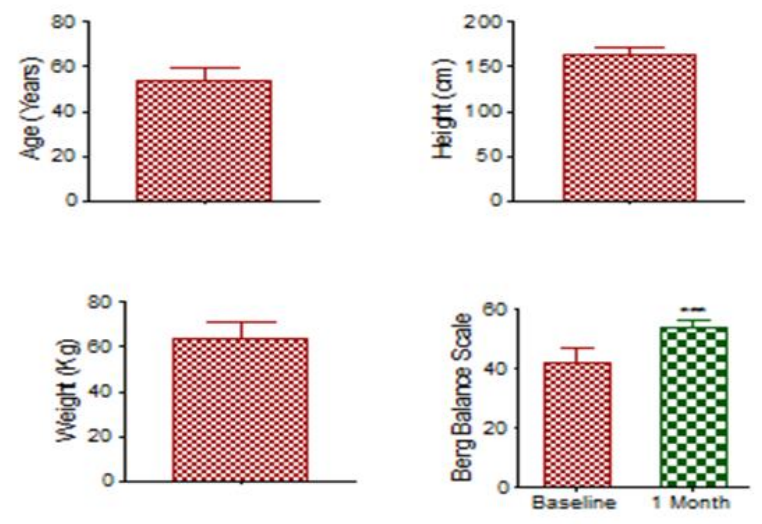

Figure 1: Demographic data and Berg Balance Scale score of the participants. ( ${ }^{* * *} \mathrm{P}<0.001$ is significant).

\section{Discussion}

The present study was undertaken to observe the effect of selected vestibular exercises on postural stability in elderly women with type 2 diabetes. There was significant increase in the postural stability followed by practicing vestibular exercises. Postural stability is common in elderly due to loss of the integration between the sensory systems [14]. Instability of gait is commonly observed in diabetes as a result of the retinopathy and neuropathy [15]. Vestibular rehabilitation was reported to be highly beneficial in the patients with dizziness and multiple sclerosis [16,17]. It was assumed that stimulation of the vestibular system through exercise triggers the sensory conflicts that cause adequate stimulation of the structures involved in the maintenance of posture and improves the stability. Further, it was reported that vestibular stimulation integrates the cortical structures through its connections and helps to overcome postural difficulties [18]. Vestibular stimulation integrates the midbrain structures like inferior colliculus, red nucleus and periaqueductal gray matter and interstitial nucleus of Cajal and maintains the balance $[19,20]$. Vestibular stimulation also integrates at the level of the spinal cord through its descending pathways [21]. Many studies have reported that lesion of the vestibular receptors bilaterally leads to the problems with balance and gait [22]. Further, interestingly, galvanic vestibular stimulation was reported to improve postural stability in patients with bilateral vestibular dysfunction and also in healthy individuals [23].

\section{Conclusion}

The present study provides further evidence for the benefitial effect of practicing the selected vestibular exercises in the management of postural stability in the elderly women with type 2 diabetes. The studies also recommend further detailed studies in this area to recommend implementation of vestibular exercises in the management of postural stability in type 2 diabetic patients in general.

\section{References}

1. Goldberg JM, Cullen KE (2011) Vestibular control of the head: possible functions of the vestibulocollic reflex. Exp Brain Res 210(3-4): 331-345.

2. Lenggenhager B, Macauda G, Grabherr L (2015) The Moving History of Vestibular Stimulation as a Therapeutic Intervention. Multisensory Res 28(5-6): 653-687.

3. Angelaki DE, Cullen KE (2008) Vestibular system: the many facets of a multimodal sense. Annu Rev Neurosci 31: 125-150.

4. Preuss N, Hasler G, Mast FW (2014) Caloric Vestibular Stimulation Modulates Affective Control and Mood. Brain Stimulat 7(1): 133-140.

5. Winter L, Kruger THC, Laurens J, Engler H, Schedlowski M, et al. (2012) Vestibular Stimulation on a Motion-Simulator Impacts on Mood States. Front Psychol 3: 499.

6. Miller SM (2016) Vestibular neuromodulation: stimulating the neural crossroads of psychiatric illness. Bipolar Disord 18(6): 539-543. 


\section{International Journal of Biochemistry \& Physiology}

7. Mittal R, Narkeesh A (2012) Review study on effect of stimulation of vestibular apparatus on postural muscle tone in cerebral palsy. J Exerc Sci Physiother 8: 11-19.

8. Massion J (1994) Postural control system. Curr Opin Neurobiol 4(6): 877-887.

9. Cenciarini M, Peterka RJ (2006) Stimulus-dependent changes in the vestibular contribution to human postural control. J Neurophysiol 95(5): 2733-2750.

10. Maeda M, Shibazaki T, Yoshida K (1979) Labyrinthine and visual inputs to the superior colliculus neurons. Progress Brain Res 50: 735-743.

11. Keshner EA, Cohen H (1989) Current concepts of the vestibular system reviewed: 1 . The role of the vestibulospinal system in postural control. Am J Occupat Ther 43(5): 320-330.

12. Ida Wiszomirska, Kaczmarczyk K, Michalina B, Andrzej W (2015) The impact of a vestibularstimulating exercise regimen on postural stability in women over 60. J Exercise Sci Fitness 13(2): 72-78.

13. Berg K, Wood-Dauphinee SL, Williams JI, Maki B (1992) Measuring balance in the elderly: validation of an instrument. Can. J. Pub. Heal 83(Suppl 2): S7-11.

14. Wiszomirska I, Katarzyna K, Michalina B, Andrzej W (2015). "The Impact of a Vestibular-Stimulating Exercise Regime on Postural Stability in People with Visual Impairment," BioMed Research Int: 8.

15. Simoneau GG, Ulbrecht JS, Derr JA, et al (1994) Postural instability in patients with diabetic sensory neuropathy. Diabetes Care 17(12): 1411-1421.

16. Badke MB, Miedaner JA, Grove CR, Shea TA, Pyle GM (2005) "Effects of vestibular and balance rehabilitation on sensory organization and dizziness handicap". Ann Otology Rhinol Laryngol 114(1): 4854 .

17. Hebert HR, Corboy JR, Manago MM, Schenkman M (2011) "Effects of vestibular rehabilitation on multiple sclerosis-related fatigue and upright postural control: a randomized controlled trial". Phy Therap 91(8): 1166-1183.

18. Deveze A, Bernard-Demanze L, Xavier F, Lavieille JP, Elziere M (2014) Vestibular compensation and vestibular rehabilitation. Current concepts and new trends. Neurophysiol Clin 44(1): 49-57.

19. Bácskai T, Székely G, Matesz C (2002). Ascending and descending projections of the lateral vestibular nucleus in the rat. Acta Biol Hung 53(1-2): 7-21.

20. Noohi F, Kinnaird C, DeDios Y, Kofman IS, Wood S, et al. (2017) Functional brain activation in response to a clinical vestibular test correlates with balance. Front Syst Neurosci 11: 11.

21. Keshner EA, Cohen H (1989) Current concepts of the vestibular system reviewed: 1 . The role of the vestibulospinal system in postural control. Am J Occupat Ther 43(5): 320-330.

22. Cullen KE (2012) The vestibular system: multimodal integration and encoding of self-motion for motor control. Trends Neurosci. 35(3): 185-196.

23. Iwasaki S, Yamamoto Y, Togo F, Kinoshita M, Yoshifuji $Y$, et al. (2014) Noisy vestibular stimulation improves body balance in bilateral vestibulopathy. Neurology 82(11): 969-975. 